\title{
Safe and Effective Digital Anticoagulation: A Continuous Iterative Improvement Approach
}

\author{
Jodie A. Austin ${ }^{1,2}$ Michael A. Barras ${ }^{3,4}$ Clair M. Sullivan ${ }^{1,5}$ \\ 1 Faculty of Medicine, University of Queensland, Brisbane, Australia \\ ${ }^{2}$ Digital Application Services ieMR, eHealth Queensland, Brisbane, \\ Australia \\ ${ }^{3}$ The School of Pharmacy, University of Queensland, Brisbane, \\ Australia \\ ${ }^{4}$ Department of Pharmacy, Princess Alexandra Hospital, Brisbane, \\ Australia \\ ${ }^{5}$ Metro North Hospital and Health Service, Brisbane, Australia
}

\begin{abstract}
Address for correspondence Jodie A. Austin, BPharm, PGDipClinPharm, The University of Queensland Faculty of Medicine, 20 Weightman Street, Herston, Queensland 4006, Australia (e-mail: jodie.austin@uqconnect.edu.au).
\end{abstract}

ACI Open 2021;5:e116-e124.

\author{
Abstract \\ Keywords \\ - electronic medical \\ record \\ - clinical decision \\ support \\ - order entry \\ - medication \\ management \\ - iterative \\ improvement \\ - anticoagulation
}

Background Anticoagulant drugs are the leading cause of medication harm in hospitals and prescribing errors are common with traditional paper prescriptions. Electronic medicines management can reduce prescribing errors for many drugs; however, little is known about the impact of e-prescribing on anticoagulants. Our case study reports on the lessons learned during conversion from paper to e-prescribing and the ongoing optimization process.

Methods The iterative implementation of an anticoagulant prescribing platform in an integrated electronic medical record (ieMR) and ongoing continuous enhancements was applied across five digital hospital sites utilizing a single domain. The collaborative management of each class of anticoagulant, optimization strategies, governance structures, and lessons learned is described. An analysis of the rate of errors and adverse events pre- and post-go live is presented.

Results The transition to e-prescribing relied on a strong inter-disciplinary governance framework to promote the safe management of anticoagulants. There was no increase in overall prescribing errors, however unfamiliarity with the new system caused a transient increase in errors with unfractionated heparin $(1.8 /$ month pre-ieMR vs. 5.5/month post-ieMR). A dedicated real-time surveillance dashboard was introduced. The iterative nature of changes indicated the complexities involved with anticoagulants and the need for an interactive, optimization approach. This led to a significant decrease in anticoagulant related hospital acquired complications (12.1/ month pre-ieMR vs. 7.8/month post-ieMR, $p=0.01$ ).

Conclusion Digitizing anticoagulant prescribing led to an overall reduction in errors, but a continuous iterative optimization approach was needed to achieve this outcome. The knowledge presented can help inform optimal therapeutic anticoagulation ieMR design strategies. received

September 18, 2020

accepted after revision

September 8, 2021
DOI https://doi.org/

$10.1055 / \mathrm{s}-0041-1736631$. ISSN 2566-9346. (c) 2021. The Author(s).

This is an open access article published by Thieme under the terms of the Creative Commons Attribution License, permitting unrestricted use, distribution, and reproduction so long as the original work is properly cited. (https://creativecommons.org/licenses/by/4.0/)

Georg Thieme Verlag KG, Rüdigerstraße 14, 70469 Stuttgart, Germany 


\section{Introduction}

Globally, the digital transformation of hospitals continues to evolve, however, most first world nations are still transitioning or maturing. In Australia there are currently only two advanced level 6/7 Healthcare Information and Management Systems Society (HIMSS) hospitals. There are six HIMSS level 6/7 sites in the United Kingdom, six in Italy, none in France, Japan or India and many other countries are only beginning the digitization of their health care systems. ${ }^{1}$ A key component to harnessing the benefits of an electronic medical record (EMR) is the implementation of an electronic medication management system (eMMS) to reduce prescribing errors. ${ }^{2}$ High-risk, complex medications are key candidates for EMR intervention strategies. Health care organisations with traditional paper-based systems use decision support tools to minimise medication risks, however, they are often inaccessible at the point of care. EMR strategies can target clinical workflows to facilitate timely decision support, laboratory results, and interdisciplinary communication.

Anticoagulants are high-risk medications with a narrow safety margin. Poor management can result in patient harm or death. ${ }^{3,4}$ They rank in the top five drug types associated with patient harm, including fatal medication incidents. ${ }^{5}$ More than $50 \%$ of anticoagulation-related adverse events are potentially preventable, ${ }^{6}$ leading the Joint Commission to include anticoagulants as part of the National Patient Safety Goal program. ${ }^{7}$ Anticoagulants are the leading cause of medication related hospital admissions ${ }^{8}$ and $8.3 \%$ of medication errors in hospitals and primary care involve anticoagulants. ${ }^{9}$ Transitions of care (admission, discharge, and surgery) pose a time of significant risk as adjustments to dose, drug, or route of administration are often necessary. ${ }^{10}$ Therefore, clear communication is essential.

Governments have committed significant investment in integrated electronic medical records (ieMR) within hospitals (\$325.2 million AUD in Queensland, Australia). ${ }^{11}$ In such systems, anticoagulants are managed using computerized physician order entry (CPOE), clinical decision support systems (CDSS), and an electronic medication administration record (MAR). CPOE and CDSS are solutions for reducing adverse drug events (ADEs) and hospital mortality rates, ${ }^{12}$ although they may introduce new types of medication errors, for example use of system generated/SIG (medical/pharmacy) abbreviations. ${ }^{13}$ Unfortunately, many system changes for anticoagulants lack supportive evidence and a recent systematic review found limited benefit demonstrated to date. CDSS alerts were most frequently studied, demonstrating varying results. Only one study evaluated the impact of dashboard implementation on the safety and quality of anticoagulant management. ${ }^{14}$

\section{Objectives}

The purpose of this case report is to describe the high-level outcomes, lessons learned, and the local strategies employed to safely manage therapeutic anticoagulation in an ieMR during implementation and the ongoing iterative improve- ment of the digital systems. Strategies for managing heparins (both unfractionated and low molecular weight), vitamin $\mathrm{K}$ antagonist (warfarin), and direct oral anticoagulants (dabigatran, apixaban, rivaroxaban) are included.

\section{Setting, Participants, Methodology, and Sequence of Events}

In Queensland, Australia, five digital hospital sites implemented a single, state-wide ieMR domain. To ensure equality, consistency, and communication between clinical and technical departments, a governance structure was established. A State-wide Medications Sub-Specialty Group was formed and met regularly to discuss current issues, potential solutions, and endorse changes for all sites across the State.

Further governance was required following a series of medications errors involving unfractionated heparin (UFH) management in the ieMR implementation phase. A Statewide Digital Heparin Working Group was formed to allow clinicians (doctors, nurses, pharmacists) and technical staff the ability to raise potential amendments to the ieMr. As issues occurred with other anticoagulants, a State-wide Digital Anticoagulation Working Group evolved (and continues to function).

\section{Audit Methodology and Statistical Analysis}

Medication errors and ADEs were identified pre and post implementation. Medication errors are reported as "incidents" by hospital staff in a database called Riskman. Data was available for four hospital sites over a 4 month pre- and 4 month post-ieMR implementation period. Any inpatient ADEs are coded and presented as hospital-acquired complications (HACs). HAC data for anticoagulants was audited for all five hospital sites over a 12 month pre- and 12 month post-implementation period. Pre and post results were compared using a Welch two sample $t$-test.

\section{Anticoagulant Management in the ieMR}

\section{Audit Results}

There was no significant difference in the mean monthly error rates for general anticoagulant medication incidents pre- and post-ieMR implementation; mean $(\mathrm{SD})=15$ (6.3)/ month vs. mean $(\mathrm{SD})=17.8$ (12.9)/month; $t(4.3) 0.38$, $p=0.72$. While a larger number of UFH infusion incidents were reported in the post-ieMR period, this did not reach statistical significance; mean $(\mathrm{SD})=1.8(1.0) /$ month, vs. mean $(\mathrm{SD})=5.5$ (3.1)/month; $t(3.6) 2.3, p=0.09$.

A retrospective audit of clinically coded bleeds (HACs) associated with anticoagulant use demonstrated a statistically significant eventual reduction in the 12 month postieMR implementation period ; mean $(\mathrm{SD})=12.1(4.4) /$ month, vs. mean $(\mathrm{SD})=7.8(3.5) /$ month; $t(21.0)-2.68, p=0.01)$.

\section{Unfractionated Heparin}

\section{Pre-Digital Management}

Prior to digitization, hospitals used a paper-based form for the prescribing, monitoring, and administration of intravenous 

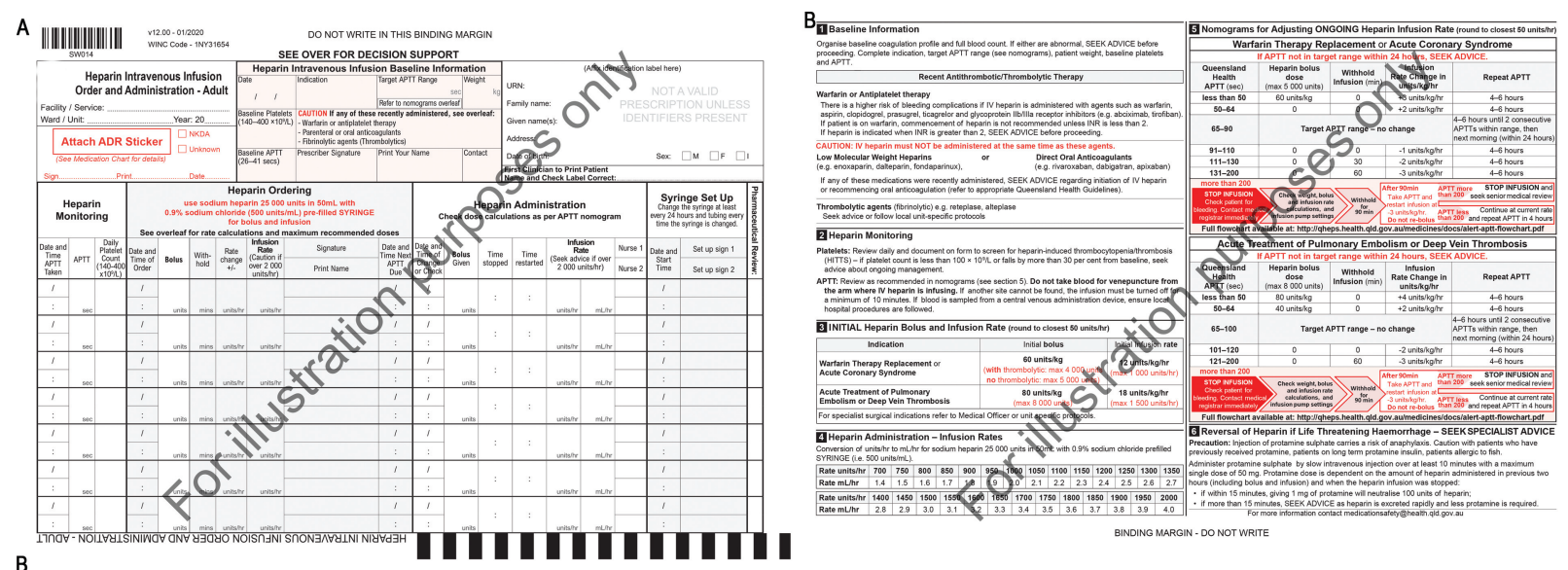

B

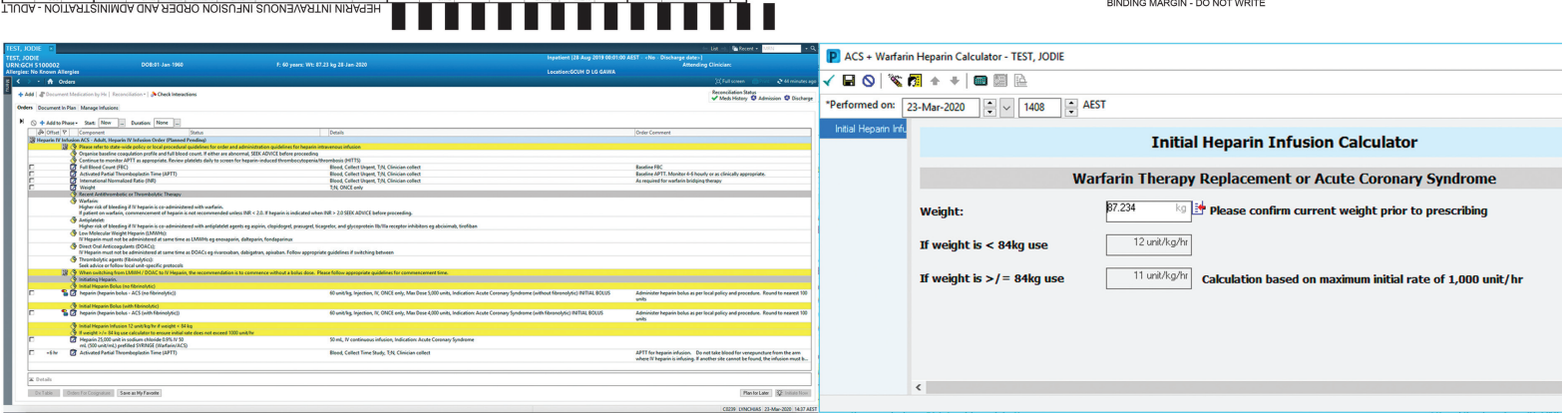

Fig. 1 (A) Paper-based form used for the monitoring, ordering, and administration of UFH (Reproduced with permission of State Government Medication Safety Committee Queensland). (B) Ordering UFH via a "Acute Coronary Syndrome" indication-based order set within the digital system.

(IV) UFH ( - Fig. 1A). Decision support was present, yet complicated and prone to error. Prescribers estimated a weightbased bolus (following consideration of the baseline activated partial thromboplastin time [aPTT]) and infusion rate (units $/ \mathrm{kg} / \mathrm{h}$ ) per indication and then converted the dose into units/h. To program a delivery rate into a syringe driver, the nurse would do an additional calculation; a $\mathrm{mL} / \mathrm{h}$ conversion. With any subsequent rate changes, the same calculations were required.

\section{Digitizing the Paper Form}

Digitizing a complex paper form was challenging and prompted a rethink of the logic and workflows for UFH. A swim-lane analysis was undertaken to convert current practice into a digital workflow to then enable future state validation by clinicians. Initially, IV UFH ordering was designed within the ieMR using indication-based order sets. Four adult-specific order sets were built to guide prescribing for; acute coronary syndrome ( - Fig. 1B), deep vein thrombosis (DVT)/pulmonary embolism (PE), bridging therapy for other anticoagulants, and low target range aPTT (for use in vascular and neurosurgery). They contained order forms, using patient's weight to calculate any weight-based conversions, reducing the complexity of multiple calculations previously performed manually. The order sets also prompted prescribers for bolus dosing, laboratory or patient care orders that should be placed concurrently with predefined offsets (i.e., delayed start times). The order sets were improved to guide the recommended course of action, dependent upon the patient's latest aPTT.

\section{Problems, Conflicts, and Constraints}

\section{Surveillance and Safety-Lessons from Incident Analysis}

The digital rollout caused a spike in reported incidents for UFH. The mean monthly rate of incidents increased from 1.8 pre-ieMR to 5.5 post-ieMR implementation $(p=0.09)$. Examples included duplicate UFH infusion orders placed or maximum recommended infusion rates being exceeded. Despite extensive training using simulation, unfamiliarity with the new system was the commonly cited reason for such incidents. To optimize safety, a dedicated surveillance process was introduced to live stream analytics using ieMR data in "an easily consumable dashboard format." ${ }^{15}$ The "Heparin Infusion Visualization Engine" (HIVE) dashboard provided "real-time" surveillance of all patients receiving UFH, flagging results to designated doctors and pharmacists, to support monitoring and patient safety. These included, time since last aPTT/platelet count, last aPTT/platelet result and the number of aPTT/platelet results in the past 24 hours. Results were color-coded to easily identify sub- versus supra-therapeutic values or time delays in routine monitoring. Platelet variance was shown to flag heparin-induced thrombocytopenia (HIT) ( $>$ Fig. 2A). Individual patient aPTT and platelet count were trended ( - Fig. 2B). Initially the HIVE dashboard was implemented to reduce the higher UFH infusion error rates during implementation, however, its use has become embedded in business as usual, in particular the cardiology service where most of the UFHs are prescribed. Pharmacy clinicians proactively monitor the dashboard 
A
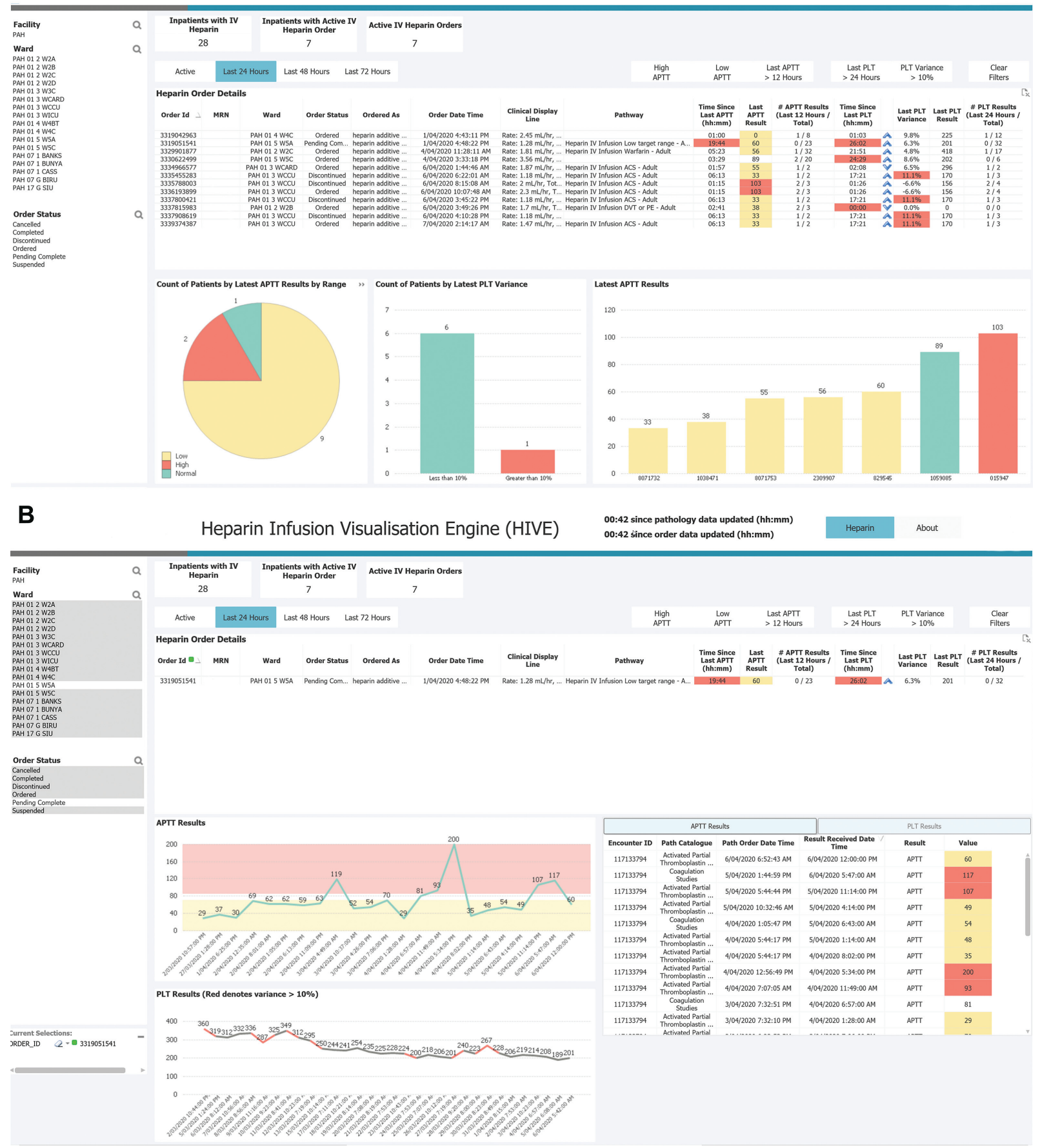

Fig. 2 (A) Heparin infusion visualization engine (HIVE) dashboard-location level view. (B) Heparin infusion visualization engine (HIVE) dashboard-patient-specific view.

regularly throughout the day and alert prescribers if action is required. A longitudinal evaluation of this dashboard is currently in progress. A systematic review of anticoagulation in digital systems found only one study addressing anticoagulant dashboard implementation with particular focus on the quality-assessment of the pharmacist verification process. $^{14}$
Continuous Improvement of the System to Ensure Patient Safety

Post ieMR implementation, modifications were made to iteratively optimize the management of UFH.

\section{Improving useability/operability}

To facilitate end-user training, in-house developed quick reference guides (written and video) which were 
uploaded onto the local intranet. Clinicians could easily access succinct dosing and monitoring information in a timely manner.

Links were embedded within order sets to give clinicians immediate access to evidence-based guidelines.

\section{Pediatric optimization}

The onboarding of tertiary pediatric hospital sites resulted in the introduction of UFH pediatric order sets for DVT/PE and for liver transplant.

\section{Iterative safety enhancements to ensure dose capping and accurate weight-based dosing}

Continuous updates were made to improve the safety of indication-based order sets. These included uplifts to enable "dose capping" on bolus doses and alerts to prescribers when exceeding a maximum weight-based dose.

To ensure an accurate dose, the order sets were designed with an order form embedded to automatically pop-up upon the prescriber initiating an infusion. The order form retrieved the patient's most recent documented weight for the current encounter (admission) and pre-populated the value to facilitate accurate units $/ \mathrm{kg} / \mathrm{h}$ dose selection. As patients may have more than one active encounter (e.g., inpatient and chronic encounter), amendments were made to search across encounters, ensuring the most recent weight pre-populated, regardless of encounter type.

The safe management of toxicity was regularly examined. Phases of the UFH order set developed for supra-therapeutic aPTT results, contained "Heparin Infusion WITHHOLD" orders. These "dummy orders" fall immediately below the continuous infusion on the MAR. They have no associated tasks, instead function to remind nurses that the UFH order is currently on hold. These orders have been built with a time limit, that moves to a discontinued status upon reaching their duration. Time limits varied with the degree of aPTT elevation (e.g., 30 minutes for aPTT 121-140 seconds vs. 60 minutes for aPTT 141-200 seconds). Physicians were notified of supratherapeutic aPTT readings and discussed with nurses withhold instructions per recommended guidelines. In this case a new "Heparin Infusion WITHHOLD 90 minutes" order was built.

Several incidents were reported where infusions had exceeded the recommended maximum rate limit. Originally, no dose range checking functionality existed due to technical constraints. A customized rule was developed, firing an alert to prescribers when the rate exceeded 40 to 45 units $/ \mathrm{kg} / \mathrm{h}$ (1,500-2,000 unit/h).

\section{Key Lessons for UFH Management}

Given the complexities associated with dosing UFH infusions, during transition from paper to digital prescribing, organizations need to be mindful of the potential rise in medication errors while staff familiarize themselves with the new system. A real-time surveillance dashboard helped to identify priority patients and summarize key elements required for UFH prescribing. Implementation of a CDSS alert was necessary to flag excessive UFH rates being prescribed, often a result of the incorrect rate unit associated with the order (units/kg/h vs. units/h).

\section{Warfarin Management}

\section{Pre-Digital Management}

Prior to ieMR, hospitals managed warfarin using a paperbased decision support tool, coupled with a dedicated prescribing and administration section on the National Inpatient Medication Chart (NIMC). ${ }^{16}$ This enabled the recording of International Normalized Ratio (INR) results and a specific administration time (-Fig. 3A). The decision support provided recommended doses, INR targets, potential drug interactions and reversal guidelines. The decision support tool was not linked to the NIMC.

\section{Digital Prescribing-An Opportunity to Combine Ordering and Decision Support}

Warfarin systems were designed using four brand and demographic specific order sets; adult versus pediatric, and Coumadin-Mylan Health Pty Ltd NSW, Australia versus Marevan-Mylan Health Pty Ltd NSW, Australia (-Fig. 3B).

The order sets were single phase, providing daily ordering of warfarin with 24 hourly offsets for each subsequent dose. A dummy "warfarin/INR dose check" order is pre-selected, populating on the MAR and prompting daily checks of warfarin orders and INR results. A "consult to pharmacy" order, also pre-selected, routed a request to Pharmacists for patient education. Laboratory orders are available for selection and drug interaction, duplication and allergy alerts exist. Links are embedded to evidence-based decision support.

\section{Problems, Conflicts, and Constraints}

\section{Continuous Improvement of the System to Ensure Patient} Safety-Key Information at Prescription

The visual field for related results was expanded to provide physicians with timely access to INR results and recent warfarin dosing, to facilitate safe and effective decisions when prescribing. This included point of care INR results to ensure all INRs, regardless of their source (laboratory collection vs. point of care) were available. These enhancements provided an effective surveillance system for patients stopping and starting warfarin (e.g., surgery), reduced dose duplication, and aided a safer prediction of future dosing.

\section{Key Lessons for Warfarin Management}

Limiting warfarin prescribing to a 24-hour duration enabled tighter control to ensure subsequent doses were appropriate for the most recent INR result. The dummy dose checks were a necessary addition to ensure required warfarin orders were not inadvertently omitted. All sources of INR results needed to be easily accessible to clinicians at the point of prescribing.

Resources were initially focused on UFH management due to the spike in user-reported incidents, however, our organization is cognisant further enhancements surrounding warfarin e-prescribing are possible and should be explored in the 
A

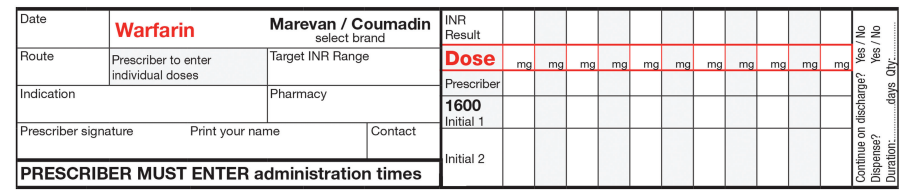

Guidelines for Anticoagulation using Warfarin - Adult

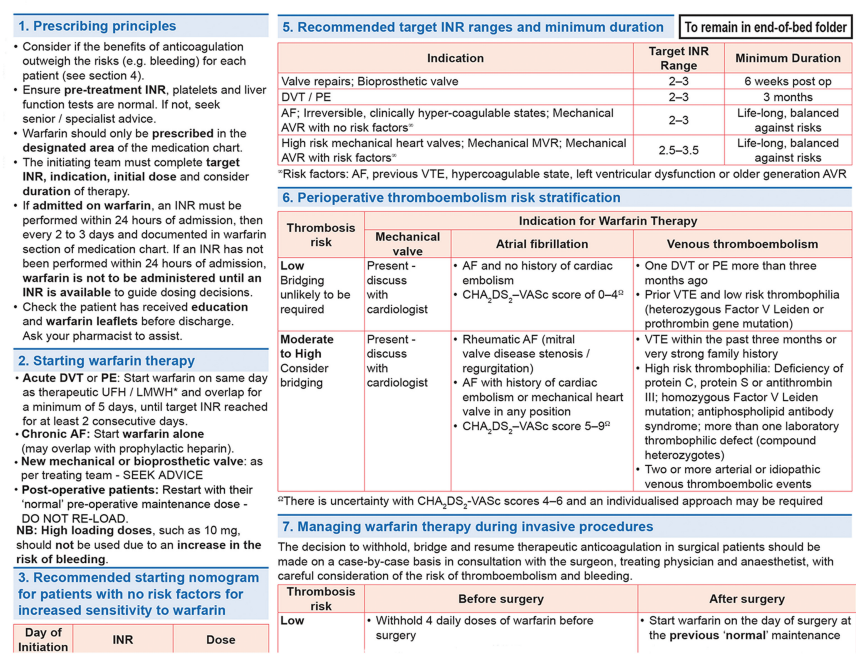

B

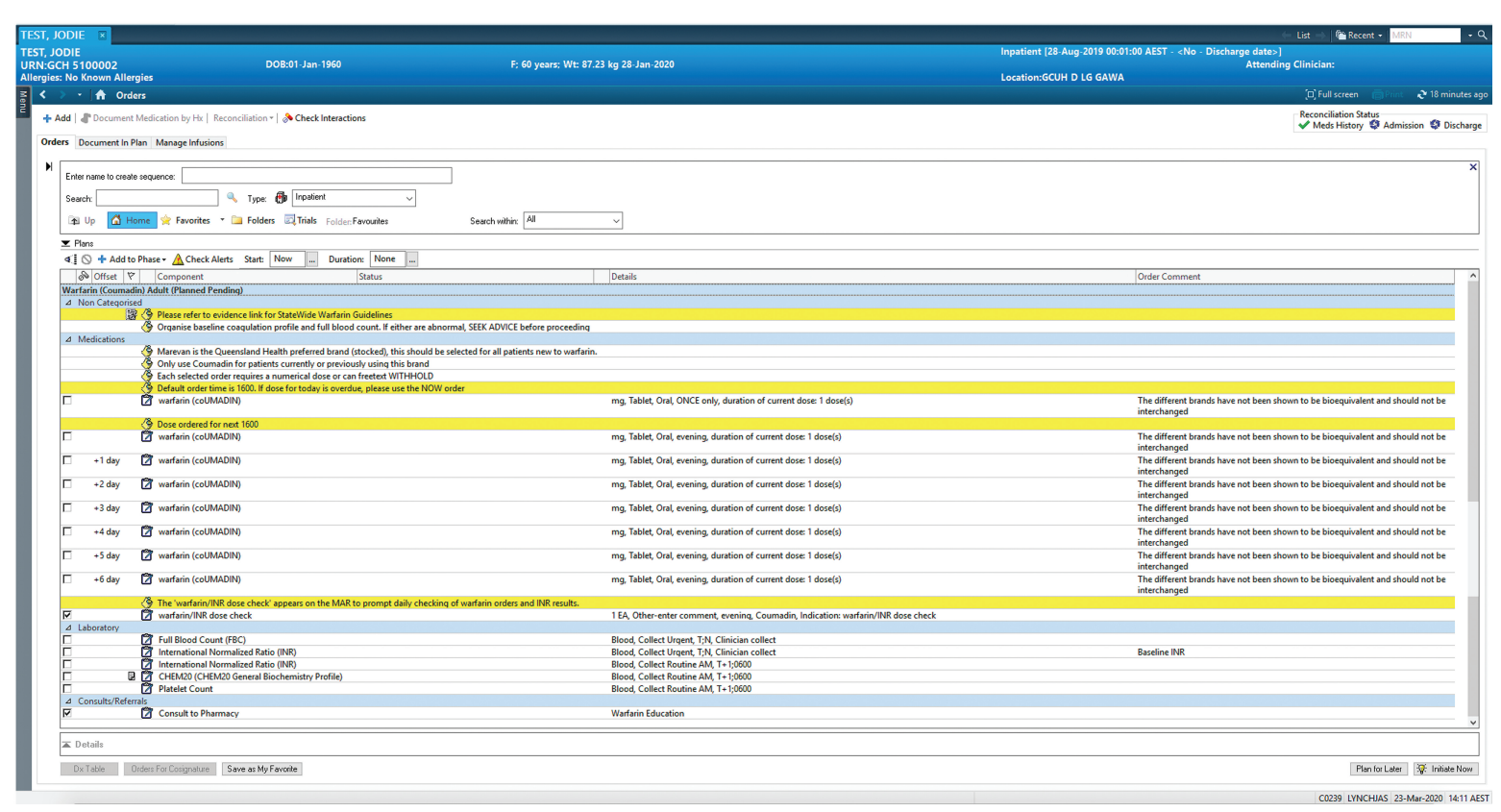

Fig. 3 (A) Ordering warfarin in a paper-based hospital (Reproduced with permission of State Government Medication Safety Committee Queensland). (B) Ordering an adult warfarin order set in a digital hospital.

future to harness addition benefits of a digital platform e.g., pharmacogenomic-guided warfarin dosing. ${ }^{17}$

4. Low-molecular weight heparins (LMWH) and directacting oral anticoagulants (DOACs) Management

\section{Pre-Digital Management}

Prescribing and administration of LMWH and DOACs prior to ieMR occurred on the NIMC, with no dedicated clinical decision support at the point of care. Local, peer reviewed guidelines existed on the State internet, however, compliance was poor. $^{18,19}$

\section{Digital Prescribing-A Focus on Patient Safety}

Both LMWHs and DOACs are available for ordering within the general order catalogue of the ieMR and drug-drug interaction, duplicate drug and drug allergy alerts will fire. Dose range checking was built, firing an alert if the dose fell outside pre-defined, licensed-dosing parameters. It is 
well known that LMWHs and DOACs require dose adjustment in patients with renal impairment. ${ }^{20,21}$ The failure to adjust dose based on renal function is a common error within an ieMR, ${ }^{22}$ therefore, customized renal rules (adult and pediatric) were developed. The use of clinical rules embedded within ieMRs has successfully demonstrated anticoagulation therapy modification in renal impairment. ${ }^{23}$ Our rules prompt alerts based upon the ordering of renally excreted medications, triggered by an estimated renal function falling below a given value ($\mathrm{mL} / \mathrm{min}$ ) (-Fig. 4A). These alerts were tailored to the drug prescribed and the degree of renal insufficiency (e.g., eGFR $<45$ for moderate-severe insufficiency specific to dabigatran prescribing, eGFR $<30$ for severe insufficiency in LMWH/DOAC prescribing). ${ }^{20,21}$
In addition, relevant laboratory results and recent drug administrations were built to display at the time of prescribing for LMWHs and DOACs ( - Fig. 4B).

\section{Problems, Conflicts, and Constraints}

Continuous Improvement of the System to Ensure Patient Safety-Standardized Dose Rounding

For practicality, it is common to round doses of LMWHs, however, impractical doses were being prescribed, particularly with the pre-built $\mathrm{mg} / \mathrm{kg}$ order sentences. For example, it is difficult to dose a $97 \mathrm{~kg}$ patient with a $97 \mathrm{mg}$ dose. While the ieMR can apply dose rounding, it was deemed undesirable given the need to accommodate different rounding increments for adult and pediatric populations. Standardized

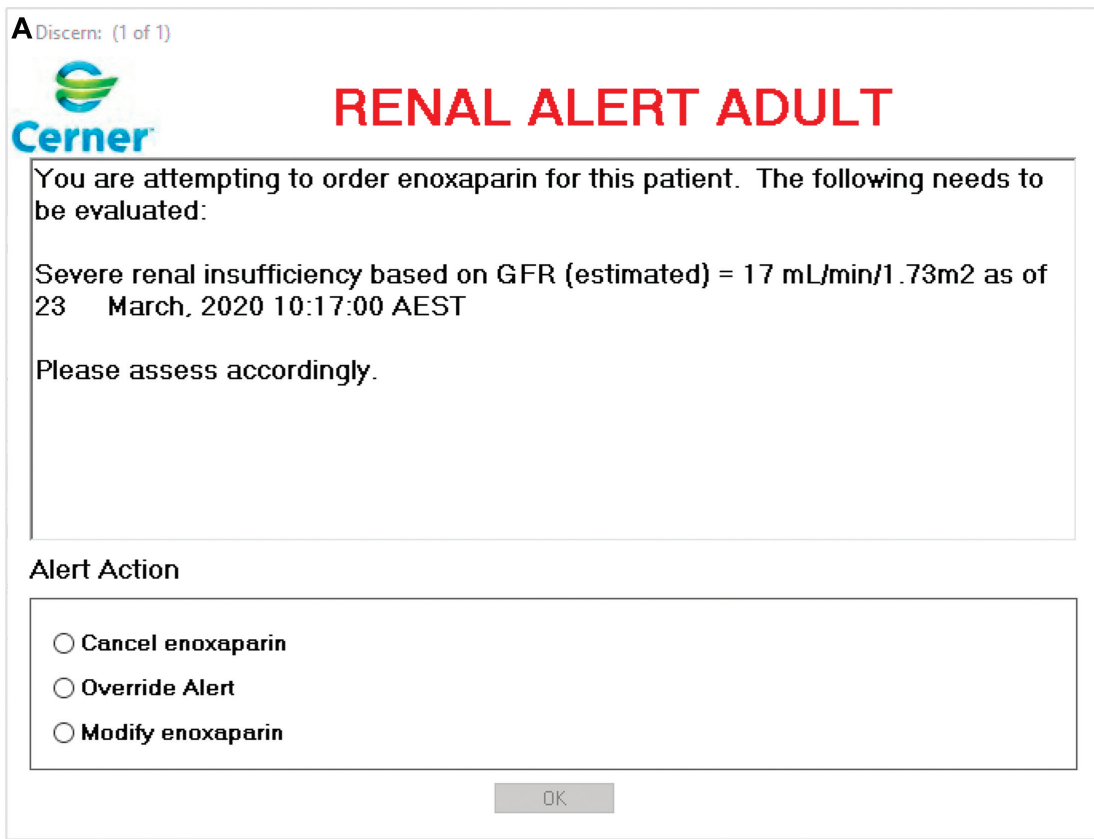

B

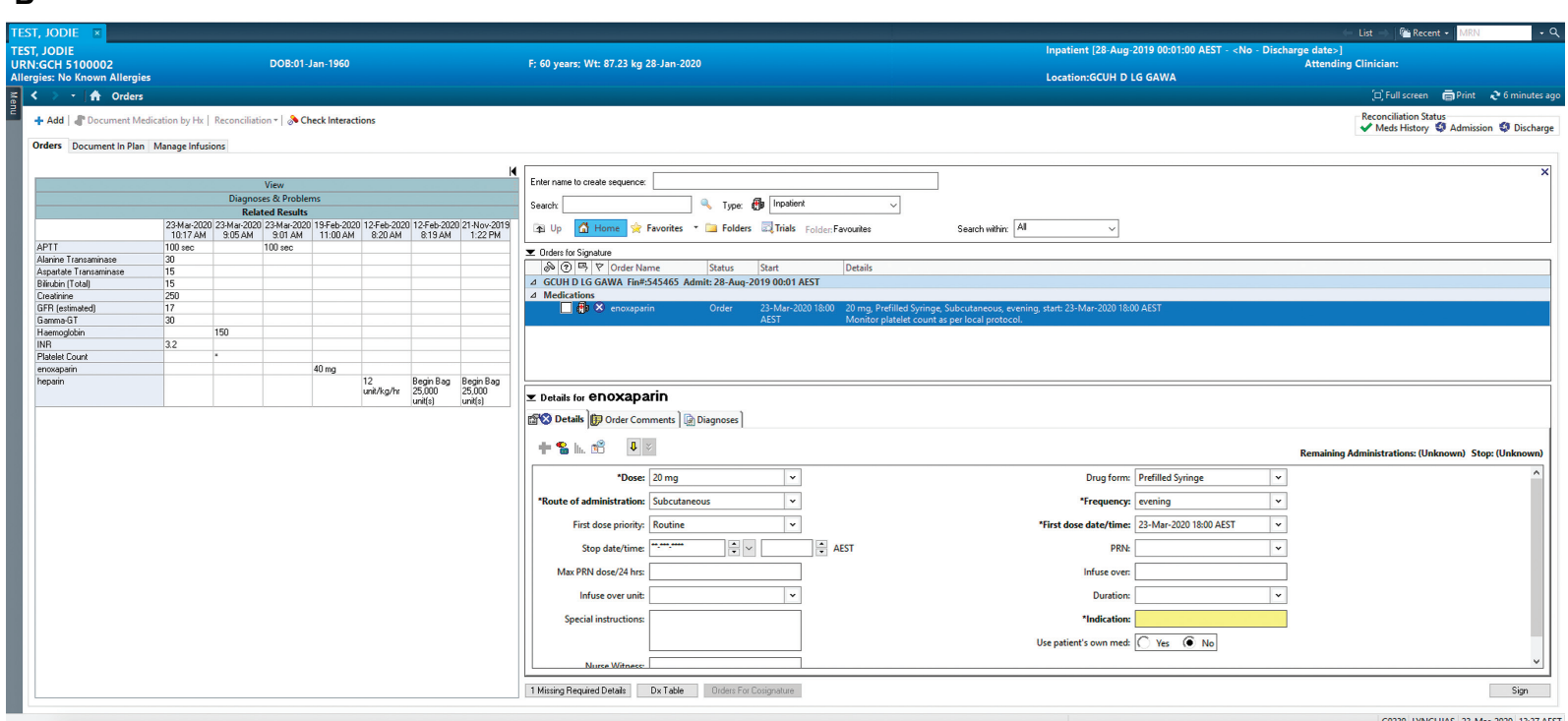

Fig. 4 (A) Example of the customized adult renal rule alert for enoxaparin. (B) Example of related results for LMWH orders-viewable on the lefthand side of the screen. 
dose banding was the accepted local solution, enabling different rounding increments based upon the calculated dose. For example, an enoxaparin dose between 19.0001 and $20.0001 \mathrm{mg}$ is standardized to $20 \mathrm{mg}$, while a dose between 95 and $105 \mathrm{mg}$ is standardized to $100 \mathrm{mg}$.

\section{Key Lessons for LMWH and DOAC Management}

Digitization provided an opportunity to embed clinical rules, prompting prescribers when potential dose adjustments were necessary in patients with impaired kidney function. When using weight-based dosing for LMWHs, consideration needs to be given to the resultant doses calculated by the digital system and additional build (e.g., standardized dose banding) may be warranted to minimize impractical doses.

\section{Discussion}

\section{Lessons Learned}

Anticoagulants are high-risk drugs. Our study showed that the digitization and continuous iterative improvement approach presented in this case study resulted in a reduction in bleeds associated with inpatient anticoagulant use.

Given the dosing complexities, the constant change in dosing guidelines, and risk of harm associated with therapeutic anticoagulation, every effort needs to be made to safely manage these drugs. Anticoagulation requires tight dose control using laboratory results such as INR, aPTT, and the patient's kidney function. For this to occur in a busy hospital environment, clinicians rely on timely and effective communication from a variety of sources. Paper-based systems have inherent deficiencies such as, locating and accessing recent results, patients' medical charts, and decision support tools. These processes are often time consuming and frustrating. Manual dose calculations and modifications are at risk of human error, in particular, weight-based dosing calculations. On a macro scale, a paper-based system is difficult to appraise and slow to optimize. The laborious nature of retrospective research such as data collection and analysis to identify issues or trends can be difficult and change management is tedious.

There are many potential benefits to the digitization of the management of anticoagulants. An ieMR requires a significant financial investment. In return, there is an expectation that systems will deliver improved patient outcomes, more efficient workflows, and the capability to access clinical data in an unprecedented manner. This case report focused on the design and implementation of a local eMMS, which at the time of publishing now supports 14 hospitals with a further 15 sites to follow. Many safety enhancements occurred, introduced on an iterative basis to allow implementation and evaluation of patient care. During the implementation we found that a clear change management process was essential and this has continued at a State level. Issues are identified, briefing papers drafted, and presented to governing bodies for endorsement. Proof of concepts are developed within non-production domains and tested by clinical staff for usability. If acceptable, such changes are then progressed to the Change Advisory Board for approval to proceed into the production domain. Changes deemed to have a significant impact on workflow are communicated with 7 days advance notice, allowing sites appropriate time to educate end users.

Under a strong governance framework substantial work has already been undertaken and the management of anticoagulants continues to evolve. For example, key leadership teams have grappled with the integration of the HIVE dashboard into clinical practice, an issue shared by others. ${ }^{24}$ As a result, a large multi-site project is underway with an aim to streamline the clinical dashboard development, governance, training and implementation process across the State. The goal is to use this framework to further expand on the HIVE dashboard, incorporating other anticoagulants to target areas of concern based upon review and analysis of key clinical metrics. Improved accessibility to these real-time and clinical analytics tools external to the ieMR appear to be the key, particularly during the early implementation phase when user-reported errors may rise.

\section{The Evidence Base Dilemma-Both Local and International}

Most of our modifications were based on peer agreed decisions, small audits of prescribing practices, and user acceptance of the digital system. We have learned many lessons, however, to date, no formal studies have been published that assess if any design decisions have reduced ADEs or improved patient safety. While this case report has presented shortterm incident and adverse event data, a large body of work is in progress to evaluate quality, safety, and costing metrics pre- and post-ieMR implementation for analysis and future publication. It was pleasing to see a statistically significant reduction in the monthly rates of clinically coded HACs associated with anticoagulant use. The anticoagulant drugs lend themselves to advanced digital techniques such as machine learning and this appears to be an exciting area of interest in the future. ${ }^{25}$

When managing therapeutic anticoagulation in an ieMR, the international literature suggests limited benefits have been demonstrated. ${ }^{14}$ Further robust research focused on patient outcomes is essential for these high-risk drugs. ${ }^{14,26}$ Health care organizations are yet to determine ideal, evidence-based-methods to enhance ieMR utilization. ${ }^{14}$ However, digital facilities are well placed to assess the impact of their interventions, consolidate findings, and improve on local design. The knowledge gained can help inform optimal ieMR design to effectively manage therapeutic anticoagulation and ultimately improve patient care.

\section{Limitations}

There are some limitations to our report. The authors acknowledge the vast number of digital systems available on an international scale, potentially limiting generalizability of certain issues and learnings presented within this case report. In addition, we recognize that most sites in the United States have already undertaken the paper to digital conversion, however, most health care systems in Asia and Europe are yet to transition and our lessons will be of benefit. The 
optimization strategies may be of benefit to established sites, in particular the HIVE dashboard.

\section{Clinical Relevance Statement}

Anticoagulants are a leading cause of medication harm in hospitals. This case report documents the implementation and optimization of an anticoagulant prescribing platform in an integrated electronic medical record (ieMR). The iterative nature of changes indicated the complexities involved with anticoagulant drugs and the need for an interactive, optimization approach. An inter-disciplinary governance framework alongside a dedicated real-time surveillance dashboard promoted the safe management of these highrisk medications.

\section{Protection of Human and Animal Subjects}

Ethics approval was sought and granted to publish this case report by the organization's Human Research Ethics Committee (Ref: LNR/2020/QMS/61139) on February 18, 2020 for low/negligible-risk research involving humans.

\section{Funding}

This research was supported by the Digital Health CRC through grant (STARS 0034).

\section{Conflict of Interest}

None declared.

\section{References}

1 Analytics HIMSS. Stage 6 \& 7 Achievement. 2017. Accessed April 05, 2021 at: https://www.himssanalytics.org/stage-6-7achievement

2 Keasberry J, Scott IA, Sullivan C, Staib A, Ashby R. Going digital: a narrative overview of the clinical and organisational impacts of eHealth technologies in hospital practice. Aust Health Rev 2017; 41(06):646-664

3 Australian Commission on Safety and Quality in Health Care. High risk medicines. 2019. Accessed April 05, 2020 at: https://www. safetyandquality.gov.au/our-work/medication-safety/high-riskmedicines/

4 Institute for Safe Medication Practices. High-alert medications in acute care settings. 2020. Accessed April 05, 2020 at: https:// www.ismp.org/recommendations/high-alert-medicationsacute-list

5 The Joint Commission. Sentinel Event Alert: Preventing errors relating to commonly used anticoagulants. 2008. Accessed April 05, 2020 at: https://www.jointcommission.org/assets/ 1/18/SEA_41.pdf

6 Sennesael AL, Larock AS, Devalet B, et al. Preventability of serious thromboembolic and bleeding events related to the use of oral anticoagulants: a prospective study. Br J Clin Pharmacol 2018;84 (07):1544-1556

7 Dager WE, Ansell J, Barnes GD, et al. "Reduce the Likelihood of Patient Harm Associated with the Use of Anticoagulant Therapy": Commentary from the Anticoagulation Forum on the Updated Joint Commission NPSG.03.05.01 Elements of Performance. Jt Comm J Qual Patient Saf 2020;46(03):173-180
8 Budnitz DS, Lovegrove MC, Shehab N, Richards CL. Emergency hospitalizations for adverse drug events in older Americans. N Engl J Med 2011;365(21):2002-2012

9 Dreijer AR, Diepstraten J, Bukkems VE, et al. Anticoagulant medication errors in hospitals and primary care: a cross-sectional study. Int J Qual Health Care 2019;31(05):346-352

10 Henriksen JN, Nielsen LP, Hellebek A, Poulsen BK. Medication errors involving anticoagulants: data from the Danish patient safety database. Pharmacol Res Perspect 2017;5(03):e00307

11 Queensland Government. Digital Projects Dashboard [cited November 26, 2020]. Available at: https://www.qld.gov.$\mathrm{au} /$ digitalprojectsdashboard/projects? offset $=1$

12 Jones SS, Heaton P, Friedberg MW, Schneider EC. Today's 'meaningful use' standard for medication orders by hospitals may save few lives; later stages may do more. Health Aff (Millwood) 2011; 30(10):2005-2012

13 Schiff GD, Amato MG, Eguale T, et al. Computerised physician order entry-related medication errors: analysis of reported errors and vulnerability testing of current systems. BMJ Qual Saf 2015; 24(04):264-271

14 Austin J, Barras M, Sullivan C. Interventions designed to improve the safety and quality of therapeutic anticoagulation in an inpatient electronic medical record. Int J Med Inform 2020; 135:104066

15 Barnett A, Winning M, Canaris S, Cleary M, Staib A, Sullivan C. Digital transformation of hospital quality and safety: real-time data for real-time action. Aust Health Rev 2019;43(06):656-661

16 Leach $\mathrm{H}$. National inpatient medication chart implementation. J Pharm Pract Res 2006;36(01):6-7

17 Melton BL, Zillich AJ, Saleem J, Russ AL, Tisdale JE, Overholser BR. Iterative development and evaluation of a pharmacogenomicguided clinical decision support system for warfarin dosing. Appl Clin Inform 2016;7(04):1088-1106

18 Dekker KR, Myers BL, Barras MA. Compliance with enoxaparin dosing and monitoring guidelines and the impact on patient length of stay: a pilot study. Ther Drug Monit 2016;38(01):59-63

19 Pattullo CS, Barras M, Tai B, McKean M, Donovan P. New oral anticoagulants: appropriateness of prescribing in real-world setting. Intern Med J 2016;46(07):812-818

20 Lim W, Dentali F, Eikelboom JW, Crowther MA. Meta-analysis: lowmolecular-weight heparin and bleeding in patients with severe renal insufficiency. Ann Intern Med 2006;144(09):673-684

21 Tran H, Joseph J, Young L, et al; Australasian Society of Thrombosis and Haemostasis. New oral anticoagulants: a practical guide on prescription, laboratory testing and peri-procedural/bleeding management. Intern Med J 2014;44(06):525-536

22 Garber A, Nowacki AS, Chaitoff A, et al. Frequency, timing, and types of medication ordering errors made by residents in the electronic medical records era. South Med J 2019;112(01):25-31

23 Ibáñez-Garcia S, Rodriguez-Gonzalez C, Escudero-Vilaplana V, et al. Development and evaluation of a clinical decision support system to improve medication safety. Appl Clin Inform 2019;10 (03):513-520

24 Waitman LR, Phillips IE, McCoy AB, et al. Adopting real-time surveillance dashboards as a component of an enterprisewide medication safety strategy. Jt Comm J Qual Patient Saf 2011;37 (07):326-332

25 Su L, Liu C, Li D, et al. Toward optimal heparin dosing by comparing multiple machine learning methods: retrospective study. JMIR Med Inform 2020;8(06):e17648

26 Sennesael AL, Krug B, Sneyers B, Spinewine A. Do computerized clinical decision support systems improve the prescribing of oral anticoagulants? A systematic review. Thromb Res 2020; $187: 79-87$ 